\title{
Medical Image Denoising and Compressing Using Discrete Orthonormal S-transform
}

\author{
Hao Huang ${ }^{1}$, Fengrong Sun ${ }^{*}$, Paul Babyn ${ }^{2}$, Zhenzhen Zhou ${ }^{1}$, Lixin Wang ${ }^{1}$ \\ 1. School of Information Science and Engineering, Shandong University, Jinan, China. \\ 2. Department of Medical Imaging, University of Saskatchewan and Saskatoon Health Region, \\ Saskatoon, Canada. \\ ('Corresponding author: sunfr@sdu.edu.cn)
}

\section{Keywords: S-transform; Huffman coding; Image denoising; Image compressing}

\begin{abstract}
S-transform is an effective time-frequency analysis technique which can provide simultaneous time and frequency distribution information similar to the wavelet transform (WT); and discrete orthonormal S-transform (DOST) can reduce the redundancy of S-transform further. So we propose a medical image denoising and compressing method using DOST. We first introduce the ideas of WT based image denoising into DOST domain and propose the thresholding-based image denoising using DOST; and then we use Huffman coding in DOST domain to compress the images. Applications for fluoroscopic image and computed tomography (CT) image illustrate the good performance of the proposed method and show its high potential for diagnostic imaging.
\end{abstract}

\section{Introduction}

S-transform is a time-frequency analysis method proposed by R. G. Stockwell in 1996 [1], it inherits and develops the localization concept of short-time Fourier transform (STFT) and continuous wavelet transform (CWT). S-transform improves the time-frequency resolution of STFT, and can be regarded as an extension or special case of wavelet transform (WT) in the multi-resolution analysis domain. Using S-transform we can get more accurate relationship between the distribution of time and frequency of the signal. To combat the redundancy caused by S-transform, R. G. Stockwell proposed the discrete orthonormal S-transform (DOST) in 2007 [2] which makes S-transform much more convenient and practical in real life.

S-transform has been applied in signal processing area successfully. For example, S-transform has been used in power quality to detect the disturbance [3] and has also been applied to diagnose the type of fault and the faulty phase on overhead transmission line [4]. Much of the research in using S-transform process medical image has examined the good performance of S-transform; e.g. some scholars used S-transform to remove the phase artifacts from fMRI data [5] and so on.

In this paper, we propose to use S-transform to transform signals from time domain to frequency domain to decrease the noise of the image and then we use Huffman coding method [6] to compress the data in DOST domain.

\section{Theory}

\subsection{S-transform}

S-transform can be regarded as an extension of continuous wavelet transform (CWT). The relationship between S-transform of function $h(t)$ and CWT is defined as:

$$
S(\tau, f)=e^{-i 2 \pi f \tau} W(\tau, d)
$$

where $W(\tau, d)$ is the generating function of CWT and has to satisfy the admissibility condition.

S-transform is also related with Fourier transform (FT). When averaged over time, S-transform becomes FT of the original signal:

$$
\int_{-\infty}^{+\infty} S(\tau, f) d \tau=H(f)
$$


where $H(f)$ stands for the FT of signals. Using this relationship between S-transform and FT, we can get the original signal:

$$
h(t)=\int_{-\infty}^{+\infty}\left\{\int_{-\infty}^{+\infty} S(\tau, f) d \tau\right\} e^{i 2 \pi f t} d f
$$

\subsection{Discrete orthonormal S-transform}

We represent S-transform with the inner product of time series $h[k]$ and the basis function $D[k]$. We use $v$ to specify the center of the frequency band, use $\beta$ to represent the bandwidth and $\tau$ to represent the time location. We can write the formula as:

$$
S_{[v, \beta, \tau]}=<D[k]_{[v, \beta, \tau]}, h[k]>=\frac{1}{\sqrt{\beta}} \sum_{k=0}^{N-1} \sum_{f=\nu-\frac{\beta}{2}}^{\frac{v+\beta}{2}-1} e^{-i 2 \pi \frac{k}{N} f} e^{i 2 \pi \frac{\tau}{\beta} f} e^{-i \pi \tau} h[k]
$$

For an $N^{*} N$ image, the number of coefficients in S-transform is $N^{2} * N^{2}$, but the number of coefficients in DOST is still $N^{*} N$ which means the redundancy and the storage requirement been reduced greatly.

We can calculate the DOST much faster by introducing the fast Fourier transform (FFT) into DOST. The formula is showed as follows:

$$
S_{(v, \beta, \tau)}=<D(k)_{(v, \beta, \tau)}, h(k)>=\frac{1}{\sqrt{\beta}} \sum_{k=0}^{N-1} \sum_{f=v-\beta / 2}^{v+\beta / 2-1} \exp \left(-i 2 \pi \frac{k}{N} f\right) \exp \left(i 2 \pi \frac{\tau}{\beta} f\right) \exp (-i \pi \tau) h(k)
$$

Using FT we can get the final formula:

$$
S_{(v, \beta, \tau)}=\frac{1}{\sqrt{\beta}} \sum_{f=v-\beta / 2}^{v+\beta / 2-1} \exp (-i \pi \tau) \exp \left(i 2 \pi \frac{\tau}{\beta} f\right) H(f)
$$

\subsection{Huffman coding}

Huffman coding is an optimal prefix coding which uses the frequency of the text to generate the Huffman tree and then use the Huffman tree to code different signals. The symbol with high frequency are allocated with short code while the symbol with low frequency are allocated with long code. The process of Huffman coding based compression can be described as follows:

1. Create a leaf node for each symbol and add it to the priority queue.

2. While there is more than one node in the queue:

1) Remove the two nodes of highest priority (lowest probability) from the queue

2) Create a new internal node with these two nodes as children and with probability equal to the sum of the two nodes' probabilities. Then add the new node to the queue.

3. The remaining node is the root node and the tree is complete.

The process of decompression is a matter of translating the stream of prefix codes to individual byte values, usually by traversing the Huffman tree node by node from the input stream.

\section{The Method of Denoising and Compressing Using Discrete Orthonormal S-transform}

\subsection{Basic Ideas}

Wavelet Transform (WT) has been widely used in image processing and has achieved successful outcome. S-transform is WT in essence which inspires us to introduce the image denoising method based on WT into S-transform domain.

Here we propose to use an appropriate threshold $\mathrm{T}$ in DOST domain to retain the original signal and restrain the noise. First we use the soft-thresholding-based image denoising strategy to process the coefficients in DOST domain. After that we find more than half of the coefficients in DOST domain becomes zero which inspires us to compress the data in DOST domain. Here we choose Huffman coding to compress the data

\subsection{Thresholding based medical image denoising}

The proposed soft-thresholding-based image denoising method can be depicted as follows:

1. Transform the noisy medical image to get its DOST coefficients $S(i, j, m, n)$.

2. Calculate the appropriate threshold $\mathrm{T}$ and let all the coefficients in DOST domain compare 
with the threshold. The equation we use to process the data can be showed as follows:

$$
S= \begin{cases}0 & a b s(S(i, j, m, n))<T \\ \frac{a b s(S(i, j, m, n))-T}{a b s(S(i, j, m, n))} S(i, j, m, n) & \text { else }\end{cases}
$$

The formula to calculate the threshold $\mathrm{T}$ is determined as in WT-based image denoising that can be depicted as:

$$
T=a \times \sigma \sqrt{2 \log (N)}
$$

where $a$ is threshold coefficient, $\sigma$ is the variance of noise and $\mathrm{N}$ is the size of the image.

\subsection{Huffman coding based medical image compression}

We code the coefficients in DOST domain after thresholding. But one problem is that the DOST domain is a plural domain which means the coefficients are plural. To decrease the complexity of coding, we separate the real part and the imaginary part of the data in processing which shows the good performance of coding. Another problem in coding is the range of the value can reach $10^{8}$ which is beyond the storage capacity of the 16 bit integer. To solve this problem, we do the linear transformation with the data and abandon the decimal part to limit it into the range of 0 65535. The result shows this method is acceptable. Then we can do Huffman coding and decoding to the different parts of the data to compress the medical-image in DOST domain. After all, we can conduct inverse DOST to get the denoised image.

\section{Clinical Applications}

\subsection{Fluoroscopic Image Denoising and Compression}

Fluoroscopic image is very important in diagnosis for the coronary heart disease, but it is seriously disturbed by noise. The previous researchers has tried different ways like using Skellam Distribution[7], self-adaptive enhancement and denoising to decrease the denoise of the images.

Here we use the data provided by Sunnybrook Health Science Centre, University of Toronto, Canada. Fig.1 and Fig.2 show the results after decompression and denoising: (a) is the original fluoroscopic image, (b) is the final image after the processing, and (c) is the error image giving the error between the original image and the decompressed image.

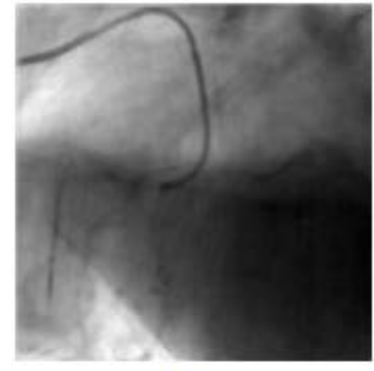

(a)

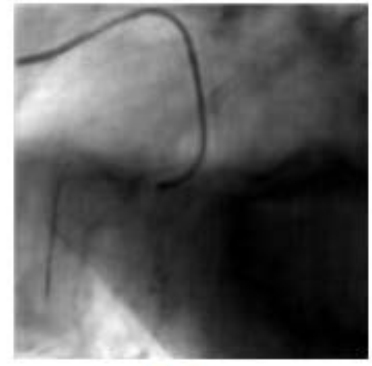

(b)

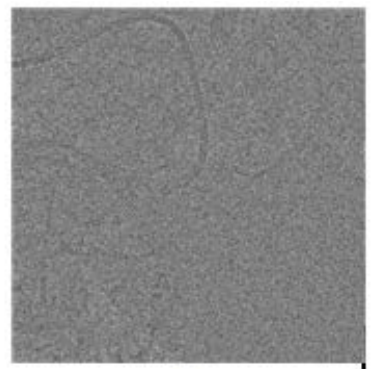

(c)

Fig.1 Denoising and decompression result for 41st frame in seq. 19th

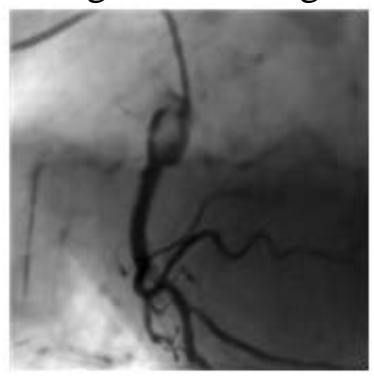

(a)

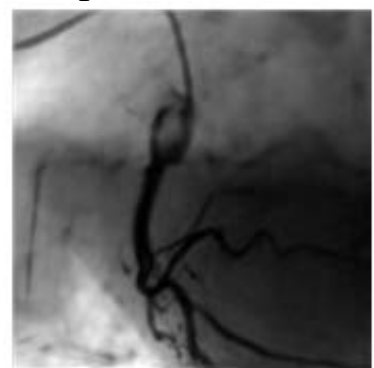

(b)

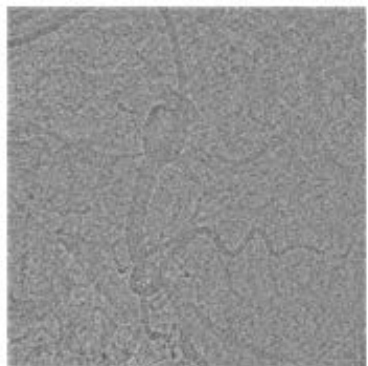

(c)

Fig.2 Denoising and decompression result of 25th frame in seq. 20th

We use SNR and CNR to evaluate the quality of the fluoroscopic image before and after processing. For the image of 41 st frame in seq. 19th, the results are as follows: 
Table 1. Quality analysis of 41st frame in seq. 19th

\begin{tabular}{|c|c|c|}
\hline & SNR & CNR \\
\hline Original image & 1.6089 & 0.6635 \\
\hline Result after denoising & 2.0574 & 1.4236 \\
\hline
\end{tabular}

For the image of 25th frame in seq. 20th, the results are as follows:

Table 2. Quality analysis of 25th frame in seq. 20th

\begin{tabular}{|c|c|c|}
\hline & SNR & CNR \\
\hline Original image & 1.1567 & 0.9235 \\
\hline Result after denoising & 1.8422 & 1.2750 \\
\hline
\end{tabular}

The threshold we use for 41st frame in seq. 19th is $T=2.4358$, the percentage of coefficients which equals to zero after thresholding is $98.24 \%$. The threshold we use in 25th frame in seq. 20th is $T=4.1767$, the percentage of coefficients equals to zero after thresholding is $97.56 \%$. The compression ratios are listed in Table 3, directly compression means we use Huffman coding directly to compress the image without any other process; and compression in DOST domain means we applied Huffman coding to the data of the image in DOST domain after thresholding.

Table 3. Compression ratio of fluoroscopic images

\begin{tabular}{|c|c|c|}
\hline & 41st frame in seq. 19th & 25th frame in seq. 20th \\
\hline Directly Compression & $13.74 \%$ & $15.91 \%$ \\
\hline Compression in DOST domain & $6.92 \%$ & $7.38 \%$ \\
\hline
\end{tabular}

\subsection{CT Image Denoising and Compression}

CT image is also widely used in current diagnostic imaging for its high quality, but the original CT image is too big to efficiently store and transmit. The previous researchers proposed to use method like lossy image compression[8], three dimensional wavelet transform [9] to compress the image. The impact of the compression on diagnostic quality also had been studied before [10].

We use the same method with what we used for fluoroscopic image. The data come from the Department of Medical Imaging, University of Saskatchewan, Canada The results are showed in Fig.3 and Fig.4: (a) is the original fluoroscopic image, (b) is the final image after processing; and (c) is the error image.

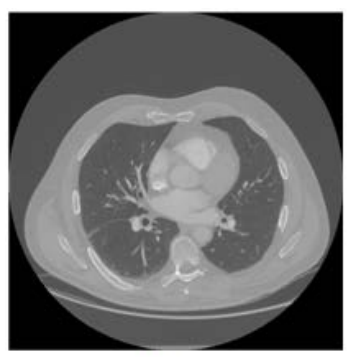

(a)

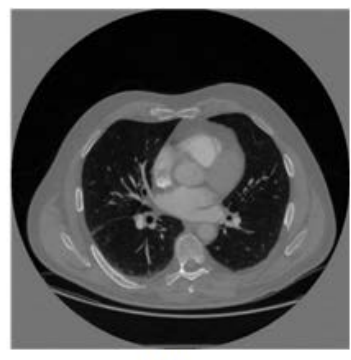

(b)

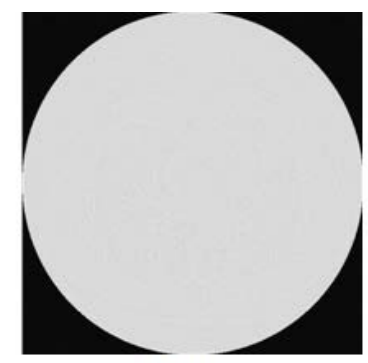

(c)

Fig.3 Decompression and Denoising result of CT image IM250

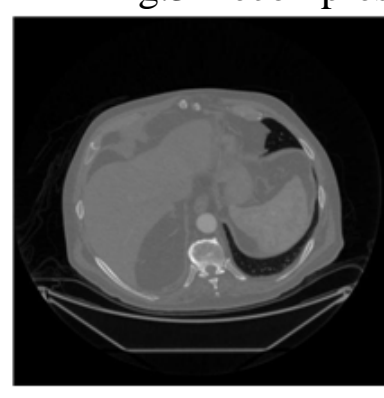

(a)

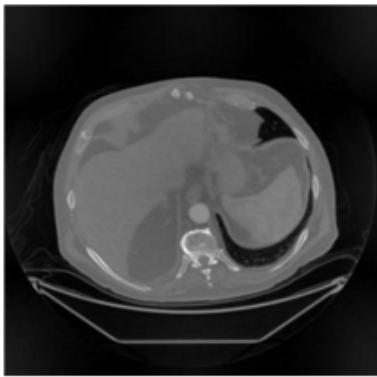

(b)

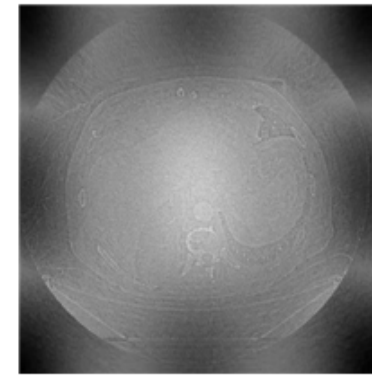

(c)

Fig.4 Decompression and Denoising result of CT image IM398

Because of the high quality of CT image, here we mainly focus on its compression. For the CT image IM250, the threshold we used in this is $T=11.3637$, the percentage of coefficients which equals to zero after thresholding is $76.86 \%$. For the CT image IM398, the threshold we used in this is $T=7.2782$, the percentage of coefficients which equals to zero after thresholding is $78.61 \%$. The Compression ratios are showed in Table 4. 
Table 4. Compression ratio of CT images

\begin{tabular}{|c|c|c|}
\hline & IM250 & IM398 \\
\hline Directly Compression & $33.18 \%$ & $29.64 \%$ \\
\hline Compression in DOST domain & $12.50 \%$ & $14.39 \%$ \\
\hline
\end{tabular}

\section{Discussion and Conclusion}

From the observation of the fluoroscopic images after denoising we can find the catheter and vessel become much clearer than the original image. The result of SNR and CNR demonstrate the quality of the image has been improved and the contrast of the catheter to the background has been enhanced. The error showed in Fig.1c and Fig.2c are caused mainly by the thresholding method. The compression ratio we get in DOST domain is less than half of the directly compression ratio in traditional domain. From the result of the CT image we can find the compression ratios we get are less than half of the directly compression ratio. At the meantime, we can remove the noise of the CT images and improve the quality of the images.

We believe that our method has achieved good results in both denoising and compression of medical images. The result of SNR and CNR guarantees the quality for quantitative analysis and the compression ratio depicts it much easier in compression of medical image.

For the further development of the method, we are now interested in solving the error caused by denoising. If we can solve this problem, the quality of the image can become much better after denoising.

Nowadays S-transform is becoming more and more popular due to the advances integrates from STFT and WT, and the fast computation from DOST. Medical image denoising is a very important part in diagnosis and analysis of medical image; and image compression is also very important in real life which will affect both using and storing of medical image. Here we combined this two part together which is time-saving and high-efficiently in denoising and compress images. Experiment results for both fluoroscopic images and CT images show the good performance of the method and its bright prospect in practical application.

\section{Acknowledgments}

This work is supported by the National Nature Science Foundation of China under the grant 61071053, and the Nature Science Foundation of Shandong Province under the grant ZR2014FM006.

\section{References}

[1] Stockwell R G, Mansinha L, Lowe R P. Localization of the complex spectrum: the S transform[J]. Signal Processing, IEEE Transactions on, 1996, 44(4): 998-1001.

[2] Stockwell R G. A basis for efficient representation of the S-transform[J]. Digital Signal Processing, 2007, 17(1): 371-393.

[3] Zhang Y, Liu Z. Application of EEMD in power quality disturbance detection[J]. Electric Power Automation Equipment, 2011, 31(12): 86-91.Zhang Y, Liu Z. Application of EEMD in power quality disturbance detection[J]. Electric Power Automation Equipment, 2011, 31(12): 86-91.

[4] Roy N, Bhattacharya K. Signal-Energy based Fault Classification of Single-Circuit Transmission Line using S-Transform and Neural Network[J]. 2014.

[5] GOODYEAR BRADLEY G, Zhu Hong-Mei, ROBERT A BROWN, et al. Removal of phase artifacts from fMRI data using a Stockwell Transform filter improves brain activity detection[J]. Magnetic Resonance in Medicine, 2004(51): 16-21.

[6] Huffman D A. A method for the construction of minimum redundancy codes[J]. Proceedings of the IRE, 1952, 40(9): 1098-1101. 
[7]Cesarelli M, Bifulco P, Cerciello T, et al. X-ray fluoroscopy noise modeling for filter design[J]. International journal of computer assisted radiology and surgery, 2013, 8(2): 269-278.

[8]Cosman P C, Davidson H C, Bergin C J, et al. Thoracic CT images: effect of lossy image compression on diagnostic accuracy[J]. Radiology, 1994, 190(2): 517-524.

[9]Wang J, Huang H K. Medical image compression by using three-dimensional wavelet transformation[J]. Medical Imaging, IEEE Transactions on, 1996, 15(4): 547-554.

[10]Koenig L, Parks E, Analoui M, et al. The impact of image compression on diagnostic quality of digital images for detection of chemically-induced periapical lesions[J]. 2014. 